\title{
ANÁLISE DA EFICIÊNCIA DA UTILIZAÇÃO DOS RECURSOS DO PROGRAMA DE AQUISIÇÃO DE ALIMENTOS MODALIDADE COMPRA COM DOAÇÃO SIMULTÂNEA
}

\author{
Murilo Didonet de Moraes ${ }^{1}$ \\ José Roberto Rambo ${ }^{2}$ \\ Omar Jorge Sabbag ${ }^{3}$
}

\begin{abstract}
RESUMO
O Programa de Aquisição de Alimentos (PAA) é uma política pública voltada à comercialização dos produtos agrícolas da agricultura familiar, implementada em 2003 no Brasil. Objetivou-se analisar a eficiência no uso dos recursos do PAA na modalidade Compra com Doação Simultânea (CDS-PAA) nos estados brasileiros participantes no ano de 2011, utilizando a metodologia DEA (Data Envelopment Analysis). $\mathrm{Na}$ análise, utilizou-se como inputs o número de agricultores familiares ocupados por estado no ano de 2006 e o número de agricultores familiares participantes do CDS-PAA por estado no ano de 2011, e como outputs utilizaram-se o número de pessoas beneficiadas pelo CDS-PAA por estado, a quantidade em quilogramas $(\mathrm{kg})$ de produtos adquiridos pela modalidade e o valor em reais $(R \$)$ dos produtos adquiridos via CDS-PAA por estado no ano de 2011. Os resultados mostraram que no uso dos recursos do CDS-PAA o Distrito Federal foi a unidade federativa que obteve a maior eficiência, enquanto o estado do Piauí apresentou a menor. Constatou-se, ainda, pela natureza dos retornos do CDS-PAA, que apenas 11 de 18 estados brasileiros atuaram em condições ótimas.
\end{abstract}

Palavras-chave: agricultura familiar, DEA, política pública.

\section{EFFICIENCY ANALYSIS OF THE USE OF RESOURCE FROM FOOD PURCHASE PROGRAMME WITH SIMULTANEOUS DONATION MODE}

\begin{abstract}
The food purchase programme (PAA) is a public policy focused on the commercialization of agricultural products implemented in the year 2003 in Brazil. This paper aimed to analyze the efficiency in the use of resources of the PAA in with Simultaneous Donation mode (CDS-PAA) by family farmers in the Brazilian states in the year 2011, using the DEA (Data Envelopment Analysis) methodology. In the

${ }^{1}$ Graduado em Agronomia (UNEMAT). Mestrado em Agronomia (FEIS/UNESP). Doutorando em Agronomia (FEIS/UNESP). E-mail: murilonx7@gmail.com

${ }^{2}$ Graduado em Agronomia (UFSM). Mestrado em Ciência do Solo (UFRGS). Doutorado em Agronomia (FEIS/UNESP). Professor da Universidade do Estado de Mato Grosso, Campus de Tangará da Serra. E-mail: jr.rambo@unemat.br

${ }^{3}$ Graduado em Agronomia (FEIS/UNESP). Mestrado em Agronomia (FEIS/UNESP). Doutorado em Geografia (FCT/UNESP). Professor da Universidade Estadual Paulista "Júlio de Mesquita Filho", Faculdade de Engenharia de Ilha Solteira. E-mail: sabbag@agr.feis.unesp.br
\end{abstract}


analysis, the number of family farmers occupied in each state in the year of 2006 and the number of family farmers participating in the CDS-PAA by state in the year 2011 were used as inputs, and as outputs the number of people who benefit from CDSPAA by state; the quantity in kilograms $(\mathrm{kg})$ of products purchased via the mode; and the value in reais $(R \$)$ of the products acquired from CDS-PAA, by state, in the year 2011. The efficiency analysis showed that the Federal District was the most efficient state in the use of resources of the CDS-PAA, while the state of Piauí, in the Northeast region, obtained the lowest efficiency. By the nature of the returns of the CDS-PAA, one could see that only 11 in 18 Brazilian states operated in optimum conditions.

Key words: Family Farming, DEA, Public Policy.

\section{INTRODUÇÃO}

Políticas públicas são diretrizes (princípios norteadores) da ação do poder público, explicitadas, sistematizadas ou formuladas em documentos como leis e programas, as quais orientam ações que normalmente envolvem aplicação de recursos públicos (TEIXEIRA, 2002). Souza (2006) completa, considerando como política pública, aquilo que em democracias estáveis, um governo faz ou deixa de fazer.

Em meados da década de 1990, emergiram políticas públicas no Brasil voltadas à construção de novas vias de desenvolvimento, atreladas, por sua vez, à agricultura familiar (DIAS et al., 2013). Tem-se como marco do surgimento de políticas públicas para a agricultura familiar brasileira o lançamento em agosto de 1995 do PLANAF - Plano Nacional de Fortalecimento da Agricultura Familiar (HESPANHOL, 2000), que culminou na implantação do PRONAF - Programa Nacional de Fortalecimento da Agricultura Familiar (LEMOS; LIMA, 2014).

$\mathrm{Na}$ sequência, o segmento agrícola familiar passou por significativas transformações, em especial, no impulso das políticas públicas (BECKER; ANJOS, 2010), fator que tem ajudado a resgatar e fortalecer a produção de produtos agrícolas tradicionais e, consequentemente, os mercados locais e regionais (DIAS et al., 2013).Como exemplo destas transformações, podemos citar a criação do Programa de Aquisição de Alimentos da Agricultura Familiar (PAA) pela Lei $\mathrm{n}^{\circ}$ 10.696 de 2 de julho de 2003 (BRASIL, 2012). Em 2003, ano da criação do PAA, foram aplicados $R \$ 144,92$ milhões, que beneficiaram 42.077 agricultores familiares, com a aquisição de 135.864 toneladas de alimentos destinados a 226.414 pessoas (PLEIN; FILIPPIN, 2012). Já em 2011, aplicaram-se $\mathrm{R} \$ 667,32$ milhões em recursos no programa, beneficiando 160.011 agricultores familiares, com a aquisição de 517.921 toneladas de alimentos destinados a 20.178 .462 pessoas (MDS, 2011). Pelas informações da Companhia Nacional de Abastecimento (CONAB), no período de 2003 a 2015, em recursos financeiros, foi aplicado no PAA o total nominal de $\mathrm{R} \$ 3,63$ bilhões que atualizados ${ }^{4}$ representariam $\mathrm{R} \$ 5,07$ bilhões (CONAB, 2016).

O PAA mostra-se também importante para a melhoria da qualidade de vida dos agricultores familiares, ao valorizar os produtos deste segmento, aproximar produtor e consumidor e ampliar a participação da agricultura familiar no mercado (PAULA et al., 2014).Embora a melhoria de vida não se restrinja ao fator renda, como apontam Sparovek et al. (2007), notou-se que os agricultores beneficiários do

\footnotetext{
${ }^{4}$ Com correção pelo IGP/DI da Fundação Getúlio Vargas de Janeiro de 2016.
} 
PAA possuíam receitas de comercialização da produção quase três vezes superiores às dos não beneficiários; fator que pode ser somado à diversificação e a qualidade da alimentação das famílias (DELGADO et al., 2005). Porém, vale observar que, segundo Aguiar e Fonseca (2014), apesar de haver uma evolução quanto às políticas públicas no meio rural brasileiro, são também enormes os desafios enfrentados para a sua implementação, fiscalização e sustentação.

Convém ainda esclarecer que a criação do PAA é resultante da confluência de dois debates importantes da década de 1990 no Brasil. O primeiro deles teve como questões centrais o combate à fome e a garantia da segurança alimentar e nutricional da população brasileira; já o segundo centralizou-se no reconhecimento da agricultura familiar como categoria social pelas políticas governamentais (GRISA et al., 2011).

De acordo com Muller et al. (2012), um dos principais objetivos do PAA é garantir a comercialização dos produtos da agricultura familiar, através do estabelecimento de preços mínimos a serem praticados com a garantia de compra, com articulação da produção com os mercados institucionais ou para formação de estoques, atendendo aos princípios da segurança alimentar. Becker e Anjos (2010) consideram que o programa estaria agindo em ações que abrangem três eixos: produção-comercialização-consumo. O PAA, desta forma, agrega no mesmo dispositivo de política pública o apoio à comercialização dos produtos da agricultura familiar e o combate à fome (MARQUES et al., 2014).

Atualmente, o PAA é executado em seis modalidades: I-Compra com Doação Simultânea; II - Compra Direta; III -Incentivo à Produção e ao Consumo de Leite; IV-Apoio à Formação de Estoques;V - Compra Institucional eVI - Aquisição de Sementes (BRASIL, 2012). A modalidade de Compra com Doação Simultânea (CDS-PAA) é a mais executada nos estados brasileiros, sendo caracterizada como a compra de alimentos diversos e a doação simultânea às entidades da rede socioassistencial, aos equipamentos públicos de alimentação e nutrição e, em condições específicas definidas pelo Grupo Gestor do PAA (GGPAA), à rede pública e filantrópica de ensino, com o objetivo de atender demandas locais de suplementação alimentar de pessoas em situação de insegurança alimentar e nutricional (BRASIL, 2012).

Assim, vê-se também que o programa possui uma relação entre três elementos: i) agricultores familiares que comercializam a produção agrícola via política pública; ii) entidade proponente, que pode ser uma cooperativa ou uma associação responsável pela intermediação entre os agricultores familiares e os consumidores e iii) entidades beneficentes que recebem os produtos agrícolas e os destinam aos consumidores finais (DEVES, 2009). Isto indica que o PAA enfoca a comercialização dos alimentos oriundos da agricultura familiar articulado com outras políticas, tais como merenda escolar, estoques de alimentos e a assistência alimentar (MULLER et al., 2012).

Chmielewska et al. (2010) observam que a modalidade CDS-PAA integra um acordo de venda e entrega prévia entre produtor e instituição beneficiária, estando fortemente ligada à criação de um circuito local de produção, comercialização e consumo, pois direciona os alimentos adquiridos para instituições cadastradas. Desta forma, a modalidade permite o surgimento de uma solidariedade em torno da alimentação, graças aos laços estabelecidos entre agricultores familiares e beneficiários, visto que os produtos destinados a doações são oferecidos para entidades assistenciais, restaurantes populares, cozinhas comunitárias e, ainda, para cestas de alimentos distribuídas pelo governo federal (PAULA et al., 2014), com efeitos em dimensões sociais, ambientais, econômicas e de produtos (MARQUES et al., 2014). 
Com base nos efeitos, importância e abrangência demonstradapelo PAA desde seu lançamento até a atualidade, entende-se que é necessário analisar sua eficiência nos estados brasileiros (na modalidade CDS-PAA), de modo que todos os recursos disponibilizados sejam aplicados da melhor forma possível para a produção de bens e serviços. Compreende-se por eficiência a combinação ótima dos insumos e métodos necessários (inputs) no processo produtivo capaz de gerar o máximo de produto (output) (PEÑA, 2008).

Neste contexto, tem-se, desenvolvida por Charnes et al. (1978), a análise envoltória de dados (DEA - Data Envelopment Analysis), uma abordagem não paramétrica ${ }^{5}$ que busca estimar a eficiência produtiva de cada unidade de produção, denominada unidade tomadora de decisão (DMU - Decision Making Unit). Esta classe de modelos, retratada por Gomes (2008), permite determinar a eficiência de uma unidade produtiva comparativamente às demais, considerando-se os múltiplos recursos de que dispõe (inputs) e os múltiplos resultados alcançados (outputs).Em outras palavras, trata-se de uma técnica baseada em programação linear com as seguintes capacidades simultâneas: a) identificar a possível fronteira de eficiência de um grupo de organizações que possuam as mesmas características; b) elaborar comparações entre os recursos usados e os resultados obtidos por cada uma das organizações avaliadas (JUBRAN, 2006).

Assim, o problema desta pesquisa busca responder à seguinte questão: dado o número de agricultores familiares ocupados e participantes do CDS-PAA por estado (inputs), qual a maior quantidade de pessoas beneficiadas, quantidade $e$ valor em reais de produtos adquiridos via CDS-PAA que se pode alcançar, mantendo-se o nível dos inputs constantes? A partir da utilização deste modelo, considera-se a hipótese de que a eficiência pode ser definida através de indicadores, de forma a mensurar a eficiência relativa dos estados, aqui tomados como DMU's, e avaliar se os mesmos estão operando com eficiência de escala, retornos crescentes ou decrescentes.

Ressalta-se ainda que a modelagem por DEA tornou-se popular na avaliação de eficiência, tanto no desenvolvimento de modelos teóricos quanto nas aplicações a casos reais (GOMES, 2008).

Para a contextualização deste trabalho, o presente estudo objetivou analisar a eficiência no uso dos recursos do CDS-PAA nos estados brasileiros participantes no ano de 2011, utilizando a metodologia DEA. Para tanto, o artigo está estruturado além desta introdução, nos seguintes tópicos: ii) materiais e métodos, apresentando os dados para confecção das variáveis a serem analisadas por DEA, respeitando-se o modelo atribuído; iii) resultados, com a discussão dos principais escores de eficiência e o retorno de escala acerca dos estados participantes e iv) conclusões, com possíveis limitações e perspectivas para trabalhos futuros.

\section{MATERIAIS E MÉTODOS}

Os dados referentes ao número de agricultores familiares ocupados foram coletados do último Censo Agropecuário e se encontram publicados no site do Instituto Brasileiro de Geografia e Estatística (IBGE, 2006).

\footnotetext{
${ }^{5}$ A vantagem de utilizar um método não paramétrico deve-se a simplificação deste em relação às exigências e pressuposições com relação aos paramétricos, viabilizando assim, pesquisas de temas relevantes para as unidades tomadoras de decisão.
} 
Todas as outras variáveis analisadas foram obtidas a partir do site da Secretaria de Avaliação e Gestão da Informação (SAGI) ligada ao Ministério do Desenvolvimento Social e Combate à Fome (MDS). A utilização do PAA (Compra com Doação Simultânea - execução CONAB - CDS-PAA) se deu em razão da quantidade expressiva de recursos e de fornecedores $(54,4 \%$ e 55,54\%, respectivamente) de tal modalidade no ano de 2011 no total de recursos e fornecedores do Programa (MDS, 2011).

Este trabalho, de natureza quantitativa, se classifica como exploratório, visando proporcionar melhor familiaridade com o problema, com vistas a torná-lo mais explícito e descritivo, no sentido de sua origem estar associada à observação, registro, análise e correlações entre fenômenos ou fatos, sem interferir no ambiente analisado. Ainda assim, houve uma abordagem qualitativa, em que o pesquisador procurou aprofundar-se na compreensão dos fenômenos que estuda. Richardson (1999) propõe que a pesquisa quantitativa é também qualitativa, pois não importa como foram efetuadas as medidas, o que é medido continua a ser uma qualidade.

Normalmente, procede-se às avaliações de eficiência por meio de funçõesfronteiras que representam uma tecnologia eficiente. Tais fronteiras são estimadas por diferentes métodos há muitos anos (MAGALHÃES; CAMPOS, 2006). Neste estudo, a determinação de fronteira eficiente foi feita mediante a técnica DEA.

De acordo com Marinho (2001), as vantagens do DEA em relação a outras técnicas de análise de eficiência se justificam pelas suas características, dentre as quais: a) a de caracterizar cada DMU como eficiente ou ineficiente através de uma única medida resumo de eficiência; b) não fazer julgamentos a priori sobre os valores das ponderações de inputs e outputs que levariam as DMU's ao melhor nível de eficiência possível; c) basear-se em observações individuais e não em valores médios; e d) permitir a observação de unidades eficientes de referência (benchmarking) para aquelas que forem assinaladas como ineficientes.

Com base nos conceitos de fronteiras eficientes da DEA para cada DMU, pode-se formular um modelo baseado em uma reta ou uma curva. No primeiro caso, tem-se o modelo que ficou conhecido como CCR, devido às iniciais dos nomes dos autores (Charnes, Cooper e Rhodes), ou também como CRS (Constant Returns to Scale). O modelo CCR permite uma avaliação objetiva da eficiência global, além de possibilitar a identificação das fontes e viabilizar estimativas de montantes das ineficiências identificadas (KASSAI, 2002). Já no segundo caso, vê-se o modelo chamado de BCC, que também possui essa denominação em razão das iniciais dos seus autores (Banker, Charnes e Cooper), conhecido ainda como VRS (Variable Returns to Scale). O modelo BCC propõe uma restrição ao CCR e possibilita a determinação da eficiência de escala, identificando assim a presença de ganhos de escala crescentes, decrescentes e constantes (KASSAI, 2002; SOUZA; WILHELM, 2009).

Os modelos DEA podem ser classificados também de acordo com o modelo de orientação desejado. Na orientação input, objetiva-se o máximo movimento em direção à fronteira a partir da redução proporcional de inputs, mantendo os outputs constantes. No modelo orientado a output, o máximo movimento rumo à fronteira é buscado a partir do acréscimo proporcional de outputs, mantendo constantes os inputs (JUBRAN, 2006; SOUZA; WILHELM, 2009).

Segundo Ali e Seiford (1993), para que haja validação da análise de eficiência, torna-se necessário que o número de unidades seja, pelos menos, duas vezes o número de insumos (inputs - $X$ ) e produtos (outputs - $Y$ ), ou seja, para um número de unidades maior que dois $(X+Y)$, sugerindo uma análise mínima para dez estados participantes do PAA no presente estudo, devendo constituir de certa homogeneidade entre eles, por utilizarem insumos semelhantes para produzirem os 
mesmos produtos, encaixando-se perfeitamente nas definições de DMU's a serem analisadas pela técnica DEA.

Neste sentido, para avaliar a eficiência no uso dos recursos do PAA nos 18 estados brasileiros participantes do CDS-PAA (Figura 1), foram utilizados como inputs o número de agricultores familiares ocupados por estado no ano de 2006 e o número de agricultores familiares participantes do CDS-PAA por estado no ano de 2011. Como outputs, consideraram-se o número de pessoas beneficiadas pelo CDSPAA por estado no ano de 2011, a quantidade em quilogramas $(\mathrm{kg})$ de produtos adquiridos via CDS-PAA por estado no ano de 2011 e o valor em reais $(R \$)$ dos produtos adquiridos via CDS-PAA por estado, também no ano de 2011.

Figura 1 - Estados brasileiros participantes do Programa de Aquisição de Alimentos da Agricultura Familiar unicamente na modalidade (CDS-PAA), 2011.

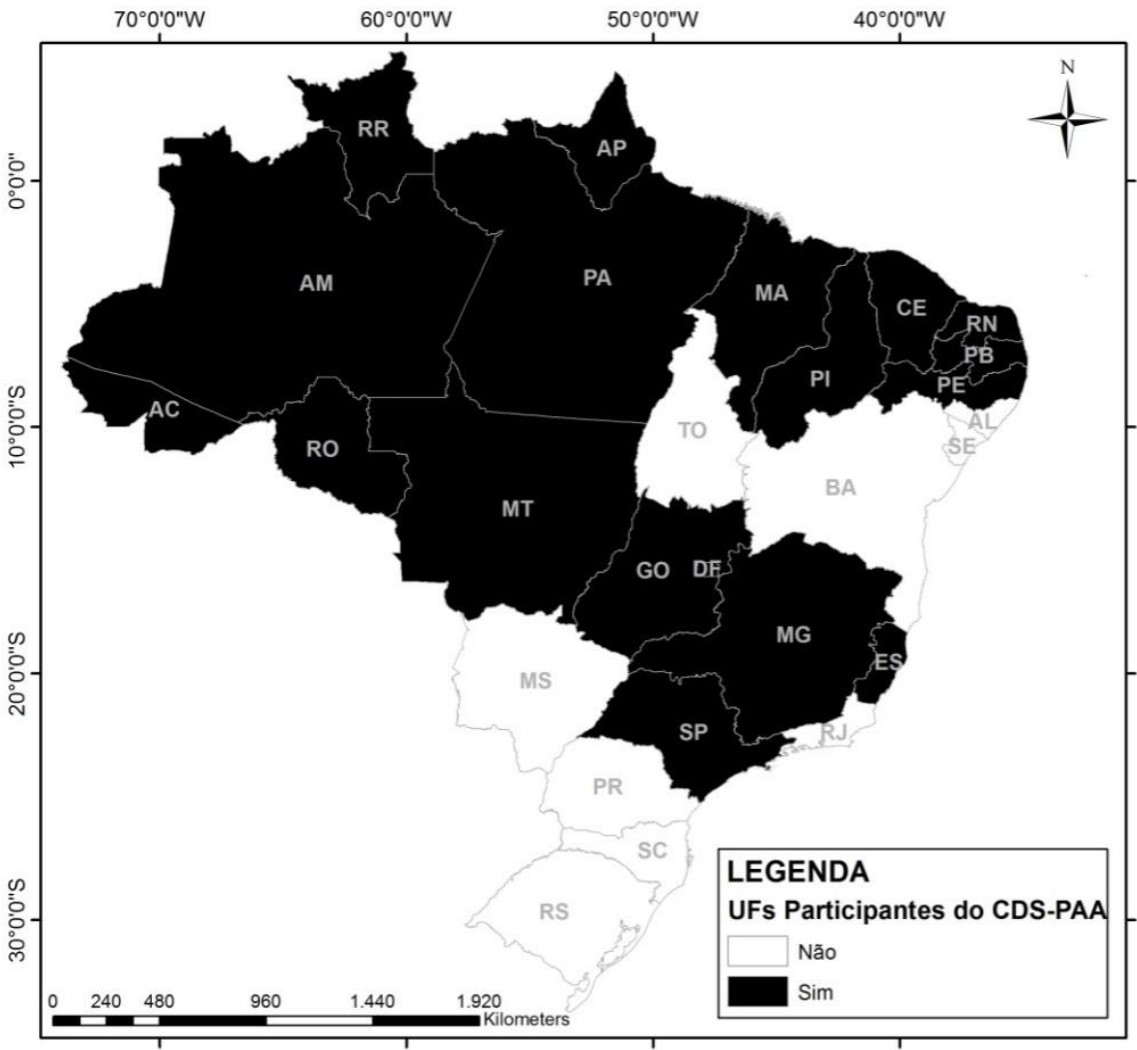

Fonte: Elaborado pelos autores.

O modelo DEA definido para a realização deste trabalho foi CCR e BCC com orientação output. Escolheu-se esta orientação pela sua melhor adequação aos objetivos do estudo, tendo em vista que as unidades avaliadas apresentam retornos variáveis de escala, isto é, o aumento dos outputs a partir da expansão dos inputs não é linear. 
forma:

A formulação matemática do modelo CCR utilizado é expressa da seguinte

$$
\max h_{o}=\sum_{j=1}^{s} u_{j} y_{j o}
$$

\section{sujeito a}

$$
\begin{aligned}
& \sum_{i=1}^{r} v_{i} x_{i o}=1 \\
& \sum_{j=1}^{s} u_{j} y_{j k}-\sum_{i=1}^{r} v_{i} x_{i k} \leq 0, \quad k=1, \ldots, n \\
& u_{j}, v_{i} \geq 0 \quad \forall i, j
\end{aligned}
$$

Em que: $h_{0}$ é a medida radial de eficiência técnica; $y_{i}$ é o valor do output $\mathrm{i} ; x_{j}$ é o valor do input j; $l_{k}$ é a importância da DMU k como referência para DMU 0 .

A formulação matemática do modelo BCC, empregado por sua vez, pode ser expressa como segue:

$$
\begin{aligned}
& \operatorname{Max} h_{0} \\
& \text { sujeito }{ }_{a}^{a}: \\
& x_{i o} \geq \sum_{k=1}^{n} x_{i k} \lambda_{i k} \forall i, \\
& h_{0} y_{j 0} \leq \sum_{k=1}^{n} y_{j k} \lambda_{k}, \forall j, \\
& \lambda_{k} \geq 0, \forall k .
\end{aligned}
$$

Em que: $h_{0}$ é a medida radial de eficiência técnica; $y_{i}$ é o valor do output $\mathrm{i} ; x_{j}$ é o valor do input j; $l_{k}$ é a importância da DMU k como referência para DMU 0.

Para a análise DEA foi utilizado o software DEAP (Data Envelopment Analysis Program), versão 2.1 (COELLI, 1996). O DEAP calcula os escores de eficiência e a natureza dos retornos que podem ser constantes, crescentes e decrescentes.

Empregou-se também o software SIAD (Sistema Integrado de Apoio à Decisão), versão 3.0 (MEZA et al., 2005) para calcular as fronteiras de eficiência padrão (quanto mais próxima da fronteira de eficiência padrão estiver a DMU, mais próximo de 1 será esse valor) e a eficiência composta normalizada (dividindo-se o 
valor da eficiência composta da DMU pelo maior valor dentre todas as eficiências compostas), a fim de se estabelecer um ranking de eficiência entre os estados brasileiros e identificar os estados referência (como critério de desempate) que servirão como benchmarking.

\section{RESULTADOS E DISCUSSÕES}

Inicialmente, os resultados mostram que os estados que utilizam unicamente o CDS-PAA possuem 7.344.780 agricultores familiares aptos a participarem do programa $(59,61 \%$ dos agricultores familiares brasileiros) (Tabela 1$)$. Deste total de agricultores familiares, 56.331 são beneficiários do programa $(35,20 \%$ do total de integrantes do PAA), atendendo a 11.846 .819 pessoas $(56,47 \%$ do total de atendidos pelo PAA), com a comercialização de $139.736 .820 \mathrm{~kg}$ de produtos agrícolas $(26,98 \%$ do total adquirido via PAA).Pode-se verificar ainda que os agricultores familiares beneficiários conseguiram auferir renda anual média pelo CDS-PAAde $\mathrm{R} \$ 4.240,25$ por agricultor familiar.

Observa-se, na comparação, entre os estados, que os valores de participação e representação do CDS-PAA são bastante diferenciados. São Paulo apresenta o maior número de pessoas beneficiadas (2.505.395 - 21,15\% do total), e o Acre, o menor (5.825 - 0,049\% do total), considerando o total de beneficiários atendidos pelo programa.

Para a produção destinada em $\mathrm{kg}$ de produtos entregues pelos agricultores familiares, o estado de São Paulo também evidencia os maiores valores, com $22,68 \%$ dos produtos disponibilizados (37.282.149 kg); em contrapartida, o Distrito Federal apresenta as menores quantidades de alimentos entregues, com 0,31\% deste total (432.716 kg). Estes estados são também os que apresentam as maiores e as menores quantidades de recursos disponibilizados pelo PAA por ano, com $19,67 \%(R \$ 46.981 .372,76)$ e $0,25 \%(R \$ 601.037,54)$ dos recursos, e de agricultores familiares beneficiados pelo CDS-PAA, com 19,90\% (11.210) e 0,24\% (137) dos agricultores familiares, respectivamente.

Já o estado de Minas Gerais é o que detém o maior número de agricultores familiares ocupados (1.177.116 - 16,05\%), e o Distrito Federal, o menor número (6481 - 0,09\%). Quando se faz a relação entre o número de agricultores familiares ocupados por estado e o número de agricultores familiares beneficiados pelo CDSPAA, São Paulo é o estado que apresenta a maior relação $(3,42 \%)$, e o estado do Piauí, a menor $(0,17 \%)$. 
Tabela 1 -Relação de Inputs e outputs avaliados na análise DEA, correspondentes aos estados brasileiros participantes do Programa de Aquisição de Alimentos da Agricultura Familiar (CDS-PAA). 2011.

\begin{tabular}{cccccc}
\hline $\begin{array}{c}\text { Estado } \\
\text { (CDS- }\end{array}$ & $\begin{array}{c}\text { Pessoas } \\
\text { Beneficiadas }\end{array}$ & $\begin{array}{c}\text { Produtos } \\
\text { Entregues em } \\
\text { kg }\end{array}$ & $\begin{array}{c}\text { Recursos } \\
\text { CDS-PAA } \\
\text { CONAB }\end{array}$ & $\begin{array}{c}\text { Agricultores } \\
\text { Beneficiários } \\
\text { CDS-PAA }\end{array}$ & $\begin{array}{c}\text { Agricultores } \\
\text { Familiares } \\
\text { Ocupados }\end{array}$ \\
\hline AC & Output 1 & Output 2 & Output 3 & Input 1 & Input 2 \\
\hline AM & 5.825 & 547.103 & $852.735,15$ & 212 & 82.889 \\
AP & 156.551 & 6.947 .740 & $7.207 .848,95$ & 1716 & 243.828 \\
CE & 556.464 & 511.174 & $1.114 .502,33$ & 292 & 10.371 \\
DF & 20.644 & 432.129 & $14.979 .870,51$ & 3.440 & 969.001 \\
ES & 132.197 & 7.382 .702 & $601.037,54$ & 137 & 6.481 \\
GO & 297.813 & 3.352 .603 & $9.052 .616,16$ & 2.355 & 202.169 \\
MA & 730.729 & 7.464 .590 & $18.331 .689,97$ & 2.050 & 212.980 \\
MG & 1.375 .463 & 23.295 .228 & $41.110 .261,62$ & 4.148 & 858.102 \\
MT & 418.498 & 6.514 .421 & $11.349 .325,27$ & 2.762 & 1.177 .116 \\
PA & 1.264 .948 & 2.895 .287 & $6.081 .112,62$ & 1.541 & 215.117 \\
PB & 1.829 .865 & 4.441 .089 & $8.570 .563,37$ & 2.019 & 665.762 \\
PE & 437.617 & 23.396 .016 & $33.335 .511,98$ & 7.532 & 710.732 \\
PI & 226.496 & 2.342 .337 & $4.566 .426,25$ & 1.195 & 722.154 \\
RN & 905.272 & 4.640 .563 & $17.353 .029,43$ & 4.176 & 191.550 \\
RO & 541.071 & 3.262 .477 & $5.765 .331,81$ & 1.411 & 233.355 \\
RR & 172.717 & 590.496 & $1.514 .543,50$ & 374 & 24.948 \\
SP & 2.505 .395 & 37.282 .149 & $46.981 .372,76$ & 11.210 & 328.177 \\
\hline Total & 11.846 .819 & 139.736 .820 & $238.857 .405,02$ & 56.331 & 7.334 .780 \\
\hline Maior & São Paulo & São Paulo & São Paulo & São Paulo & Minas Gerais \\
\hline Menor & Acre & Distrito & Distrito Federal & Distrito & Distrito \\
& & Federal & & Federal & Federal \\
\hline Fon & & & &
\end{tabular}

Fonte: MDS (2011) e IBGE (2006).

Esta diferença entre os estados de São Paulo e Piauí pode ser explicada a partir dos comentários de Sagrilo et al. (2002) e Sant'Ana et al. (2013). No Piauí, tem-se, de acordo com Sagrilo et al. (2002), uma agricultura familiar local caracterizada por baixo poder aquisitivo, acesso limitado às tecnologias (sem utilização de insumos, poucos cuidados dedicados a animais), além de baixos níveis educacionais $(68,7 \%$ dos chefes de família e $63,7 \%$ das donas de casa são analfabetos). Sant'Ana et al. (2013), por sua vez, afirmam que no noroeste de São Paulo, apesar de os agricultores familiares também apresentarem baixos níveis educacionais, os mesmos possuem diversificação de atividades agropecuárias e acesso a recursos tecnológicos e de formação voltados ao processamento de produtos agrícolas, estando ainda intimamente inseridos na lógica de comercialização de seus produtos com o mercado varejista ou o consumidor final.

Merece destaque também que, tendo como base a eficiência padrão (Tabela 2), nove dos 18estados brasileiros estudados são categorizados como eficientes. Porém, este tipo de discriminação não fornece as bases para afirmar qual destes estados é o mais eficiente. Por isso, recorreu-se aos valores da eficiência composta normalizada como critério de desempate entre os estados eficientes, no intuito de identificar o estado mais eficiente, que servisse debenchmarking para os estados com menor eficiência. 
Tabela 2 - Valores e ranking de eficiência no uso dos recursos por parte dos agricultores familiares nos estados brasileiros participantes do DS-PAA em 2011.

\begin{tabular}{|c|c|c|c|}
\hline Posição & Estado (DS-PAA) & Eficiência Padrão & $\begin{array}{c}\text { Eficiência Composta } \\
\text { Normalizada }\end{array}$ \\
\hline 10 & DF & 1,000000 & 1,000000 \\
\hline $2^{\circ}$ & PB & 1,000000 & 0,988663 \\
\hline $3^{\circ}$ & PE & 1,000000 & 0,986513 \\
\hline $4^{\circ}$ & SP & 1,000000 & 0,977626 \\
\hline $5^{0}$ & MA & 0,999078 & 0,967550 \\
\hline $6^{\circ}$ & AM & 1,000000 & 0,967178 \\
\hline $7^{\circ} \underline{0}$ & GO & 1,000000 & 0,936841 \\
\hline $8^{\circ}$ & ES & 0,972808 & 0,932269 \\
\hline $9^{0}$ & MG & 0,953512 & 0,924087 \\
\hline $10^{\circ}$ & $\mathrm{RR}$ & 0,991360 & 0,915623 \\
\hline $11^{\circ}$ & RO & 0,937968 & 0,901275 \\
\hline $12^{0}$ & $A C$ & 1,000000 & 0,898334 \\
\hline $13^{\circ}$ & AP & 1,000000 & 0,898334 \\
\hline $14^{\circ}$ & CE & 1,000000 & 0,898334 \\
\hline $15^{\circ}$ & MT & 0,933264 & 0,889000 \\
\hline $16^{\circ}$ & $\mathrm{RN}$ & 0,969592 & 0,871017 \\
\hline $17^{\circ}$ & PA & 0,928895 & 0,855739 \\
\hline $18^{\circ}$ & $\mathrm{PI}$ & 0,867537 & 0,779338 \\
\hline
\end{tabular}

Fonte: Dados da pesquisa.

A partir disso, pode-se inferir que o Distrito Federal é a unidade federativa mais eficiente no uso dos recursos do CDS-PAA. As causas que fizeram com que o Distrito Federal assumisse tal posição podem estar relacionadas ao seu equilíbrio frente aos inputs e outputs analisados, pois embora tenha o menor número de agricultores participantes do PAA, receba a menor porção de recursos e adquira uma menor quantidade de produtos, o Distrito Federal beneficia mais pessoas do que o estado do Acre, por exemplo, o qual, por sua vez, tem mais agricultores e uma maior quantidade de recursos. Em outras palavras, o Distrito Federal consegue beneficiar uma maior quantidade de pessoas (outputs), mesmo com um menor contingente de pessoas e uma menor quantidade de recursos (inputs).

Segundo Valnier e Ricci (2013), a operacionalização das modalidades do PAA depende muito das características de cada região, como o tipo de agricultura desenvolvida, as distâncias dos centros consumidores, as dificuldades de acesso e, principalmente, a cultura organizacional dos agricultores familiares. Os pontos levantados pelos autores podem justificar a eficiência do Distrito Federal, pois no estado, os agricultores familiares possuem: i) proximidade de grandes centros consumidores; ii) boa infraestrutura viária; iii) agência de assistência técnica e extensão rural (ATER) efetiva; iv) acesso à informação; v) incentivo à produção orgânica (de maior valor agregado); vi) diversificação da produção e vii) ocupação territorial (CORREIO BRAZILIENSE, 2014).

Por outro lado, o estado do Piauí demonstra a menor eficiência no uso dos recursos do CDS-PAA. Entre as características que explicam tal posição, podem estar relacionados à precariedade no estado de: i) infraestrutura viária; ii) serviço de ATER; iii) acesso à informação e iv) estrutura de mercado. Além disso, deve-se relembrar a baixa relação entre o número de agricultores beneficiários do CDS-PAA e o número de agricultores familiares no estado, característica que pode estar associada à divulgação restrita do Programa, impedindo, desta forma, que um número maior de pessoas tenha acesso ao PAA. Um dos motivos desta divulgação limitada pode estar associado a uma preocupação dos gestores no que diz respeito 
ao descompasso existente entre o crescimento do número de agricultores que buscam acessar o PAA e sua oferta orçamentária (GRISA et al., 2010).

$\mathrm{Na}$ Tabela 3, quando se analisam os escores de eficiência de escala, o estado do Acre é o único que apresenta natureza de retornos crescente. Porém, pode ser considerada uma DMU falso eficiente, já que esta unidade de federação,comparada com o Distrito Federal, tem número mais expressivo de agricultores familiares beneficiários do CDS-PAA, mais recursos destinados ao programa por estado/ano, bem como maior quantidade de produtos comercializados; apesar disso, é muito menor o número de pessoas beneficiadas, conforme se expôs na Tabela 1. E quando se compara a relação entre o número de pessoas beneficiadas e o número de agricultores beneficiários do CDS-PAA (Tabela 1), o Distrito Federal atinge um valor de 150,69 pessoas beneficiadas por agricultor beneficiário, enquanto o Acre apresenta somente 27,48 pessoas beneficiadas por agricultor.

Segundo Andrade et al. (2015), este fato pode ser explicado pela heterogeneidade entre os Estados, associada a um profundo desnível socioeconômico e as diferentes interações humanas, os quais refletem nas realidades encontradas, diferenciando os resultados da implementação e da operacionalização das políticas públicas de caráter universal. Assim, essas distintas realidades são responsáveis pela dificuldade encontrada pelos agricultores familiares para acessar os recursos existentes, principalmente nas regiões mais carentes, como o norte e nordeste do país. 
Tabela 3 - Escores de eficiência de escala e natureza dos retornos dos estados brasileirosparticipantes do CDS-PAA em 2011.

\begin{tabular}{ccc}
\hline Estado (CDS-PAA) & Eficiência de Escala & Natureza dos Retornos \\
\hline AC & 0,995 & Crescente \\
AM & 1,000 & Constante \\
AP & 1,000 & Constante \\
CE & 0,999 & Decrescente \\
DF & 1,000 & Constante \\
ES & 0,994 & Decrescente \\
GO & 1,000 & Constante \\
MA & 0,999 & Decrescente \\
MG & 0,965 & Decrescente \\
MT & 0,987 & Decrescente \\
PA & 1,000 & Constante \\
PB & 1,000 & Constante \\
PE & 1,000 & Constante \\
PI & 1,000 & Constante \\
RN & 0,989 & Decrescente \\
RO & 1,000 & Constante \\
RR & 1,000 & Constante \\
SP & 1,000 & Constante \\
\hline
\end{tabular}

Fonte: Dados da pesquisa.

Percebe-se, ao mesmo tempo, que 11 dos 18 estados brasileiros são verdadeiramente eficientes, operando em eficiência de escala com retornos de natureza constante (Figura 2). Isso se justifica por um equilíbrio entre outputs e inputs, o que sugere que os estados estão operando nas suas capacidades ótimas. Em outras palavras, os agricultores familiares participantes do CDS-PAA estão comercializando uma quantidade expressiva de produtos, com um importante valor agregado e beneficiando muitas pessoas. Não seria razoável, portanto, reduzir a quantidade de recursos do CDS-PAA para esses estados por estarem operando em escala ótima. Pelo contrário, deve-se buscar, sistematicamente, a ampliação dos recursos para o programa, a partir do estreitamento da razão entre o número de agricultores familiares ocupados e os participantes do CDS-PAA, de modo a expandir ainda mais seus benefícios.

Além disso, os estados que evidenciam retornos de natureza constante revelam os níveis ótimos ou targets (metas) de operacionalização das variáveis analisadas. Esses targets- presentes nas experiências dos agricultores, nos investimentos realizados pelos governos estaduais e no apoio das mais diversas entidades - podem servir como referências ou benchmarks para a projeção de outros estados ineficientes em direção à fronteira da eficiência.

Dentro deste contexto, Camargo et al. (2013) apresentam uma característica importante do estado de São Paulo que pode servir como referência a outros estados. Geralmente, as políticas para a agricultura familiar costumam ser formuladas e executadas pelos governos federal e estadual apenas, recebendo pouca adesão das prefeituras municipais. Contudo, o PAA provocou uma mudança nesta tendência, de modo que nos municípios onde em que há a implantação do CDS-PAA executadas pelas prefeituras, sucede uma aproximação entre a gestão municipal e a agricultura familiar. Isto pode ser observado no município de Suzano, onde a aplicação do PAA culminou na vinda de outros programas municipais para os agricultores, como a Patrulha Rural, serviço de ATER e feira de produtores.

Por outro lado, têm-se seis dos 18 estados brasileiros operando em retornos de natureza decrescente. Desta forma, pela quantidade de recursos 
destinados ao CDS-PAA por ano, esses estados comercializam uma quantidade pequena de produtos, apesar de atender a uma grande quantidade de beneficiários. Algumas possibilidades para que esta situação seja revertida seria diminuir os recursos destinados ao CDS-PAA nesses estados para adquirir a mesma quantidade de produtos, ou melhorar o nível tecnológico do sistema de produção agrícola dos agricultores familiares. E seguindo o que comentam Delgado et al. (2005), o caso CDS-PAA precisaria de mecanismos eficientes de controle e de fiscalização, em especial, para esses estados.

Em particular para os estados do Ceará, Maranhão e Rio Grande do Norte, os resultados podem ser expressos devido a alguns desajustes, tais como o uso político do PAA, a demora no recebimento do pagamento até que o produto fosse entregue na CONAB, bem como a compra por atravessadores ou agricultores patronais das DAP's dos agricultores familiares para poderem vender ao PAA (MDS, 2006).

Figura 2 - Estados brasileiros participantes do Programa de Aquisição de Alimentos da Agricultura Familiar unicamente na modalidade (CDS-PAA) e a natureza de retornos em 2011.

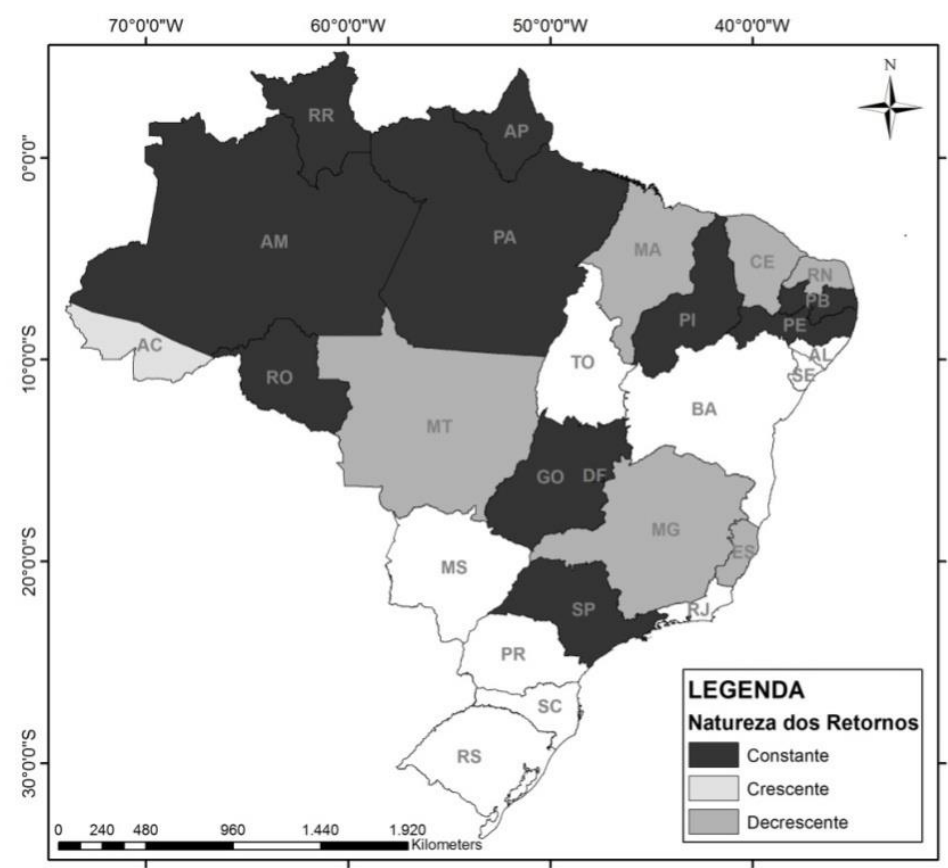

Fonte: Elaborado pelos autores.

Ressalta-se que,por meio do PAA, se estabelece a formação de um mercado institucional específico, no qual paira certa garantia de venda da produção a preços que buscam valorizar o trabalho do agricultor familiar sem as oscilações verificadas normalmente no mercado. Neste sentido, apesar de São Paulo possuir um nível ótimo de operação constatado no presente estudo, problemas como de infraestrutura, transporte da produção, assistência técnica e orientação produtiva limitam a potencialidade da produção agrícola familiar em alguns municípios da 
região Sudeste (LOPES; ALMEIDA, 2012).Nas regiões mais pobres do país, exemplos do Norte e Nordeste, esta situação tende a ser mais impactante.

Em Minas Gerais, estado que apresenta o menor valor de eficiência relativa $(96,5 \%)$, observa-se que apesar da maioria dos recursos públicos destinados ao PAA estar focalizada junto ao público demandante de suas ações, sua taxa de cobertura nessas regiões ainda é muito incipiente, dada às necessidades locais dos municípios, podendo ser ampliadas no estado (SIMÃO et al., 2014).

Convém destacar que em políticas públicas, o monitoramento e a avaliação de programas (como o PAA), contemplando a avaliação dos processos e da eficiência das ações, apontam caminhos e soluções, além de ser realizada com o intuito de fundamentar a tomada de decisão e aprimorar os processos de gestão (MONTEIRO, 2002). Assim, aspectos relacionados à capacidade de gestão de políticas públicas podem e devem ser otimizados para aumentar a eficiência e a governança do governo (PEDROSO et al., 2009).

Por fim, o conceito de eficiência, por meio da análise envoltória de dados, aplicada a programas existentes dentro de uma política voltada ao segmento rural, está relacionada à ideia de possibilidades de trabalho em termos de geração de produtos e de configuração de resultados alcançáveis com os recursos disponíveis e os insumos utilizados, comparativamente a uma fronteira de desempenho possível de ser alcançada (REIS, 2012). Em consonância, Belloni (2000) infere que o critério de eficiência está associado ao conceito de racionalidade econômica, revelando ainda a capacidade da organização - neste caso os estados da federação - de produzirem um máximo de resultados com o mínimo de recursos disponíveis para tal.

\section{CONCLUSÕES}

Conforme os resultados obtidos, verificou-se que o CDS-PAA no estado de São Paulo possui o maior número de pessoas beneficiadas, maior comercialização de produtos, maior quantidade de recursos destinados por estado/ano e o maior número de agricultores familiares beneficiários.

Por outro lado, com base nos valores da eficiência composta normalizada, observou-se que o Distrito Federal foi a unidade federativa mais eficiente no uso dos recursos do CDS-PAA, enquanto o estado do Piauí obteve a menor eficiência.

Do ponto de vista da natureza dos retornos, apenas 11 dos 18 estados brasileiros estão operando em condições ótimas, isto é, apresentam equilíbrio entre outputs e inputs. Isto significa que os recursos do CDS-PAA aplicados nesses estados estão beneficiando um contingente significativo de pessoas e adquirindo uma quantidade expressiva de produtos. Compreende-se que a experiência desses estados pode servir como referência para estados ineficientes.

Vale ressaltar, ainda, que a análise DEA demonstrou ser uma ferramenta que pode auxiliar as esferas governamentais no aperfeiçoamento do programa CDSPAA, pois as distintas experiências apontam os aspectos que devem ser aprimorados e/ou incentivados.

Cabe destacar que uma possível limitação das análises de eficiência seja atribuída à dificuldade de aceitação por parte dos gestores, sem a devida formação específica, que pode ser atenuada por capacitação gerencial.

Por outro lado, existe a possibilidade em futuras pesquisas sobre as principais causas determinantes da ineficiência, por meio da aplicação de técnicas econométricas que incorporam novas variáveis não limitadas apenas aos escores de eficiência determinados pela análise DEA, no tocante aos recursos destinados pelo PAA na modalidade Compra com Doação Simultânea para os estados participantes. 


\section{REFERÊNCIAS}

AGUIAR, C. C.; FONSECA, B. C. Evolução, acesso e efetividade das políticas públicas para a agricultura familiar no governo Lula: um estudo do Conselho Municipal de Desenvolvimento Rural Sustentável de Viçosa, Minas Gerais. Extensão Rural, Santa Maria, v. 21, n. 3, p. 121-145, 2014. Disponível em: $<$ https://periodicos.ufsm.br/extensaorural/article/view/7854>. Acesso em: 19 out. 2016.

ALI, A. I., SEIFORD, L. M. The Mathematical Programming Approach to Efficiency Analysis. In: FRIED, H. O., LOVELL, C. A. K., SCHIMIDT, S. S. (Orgs.). The Measurement of Productive Efficiency: Techniques and Application. New York: Oxford University Press, 1993. p. 120-159.

ANDRADE, A. A. X.; SILVA, G. B.; ANDRADE, N. A. X. O acesso às políticas públicas no semiárido e seu reflexo na heterogeneidade da agricultura familiar: o PNAE em Boquira-BA. Extensão Rural, Santa Maria, v. 22, n. 2, p. 79-97, 2015. Disponível em: <https://periodicos.ufsm.br/extensaorural/article/view/15443>. Acesso em: 18 out. 2016.

BECKER, C.; ANJOS, F. S. Segurança alimentar e desenvolvimento rural: limites e possibilidades de aquisição de alimentos da agricultura familiar, em municípios do sul gaúcho. Segurança Alimentar e Nutricional, Campinas, v. 17, n. 1, p. 61-72, 2010.

BELLONI, J. A. Uma metodologia de avaliação da eficiência produtiva de Universidades Federais Brasileiras. Tese (Doutorado) - Programa de PósGraduação em Engenharia de Produção, do Departamento de Engenharia de Produção e Sistemas, da Universidade Federal de Santa Catarina. Florianópolis: UFSC, 2000.

BRASIL. Decreto n 7.775, de 4 de julho de 2012. Regulamenta o art. 19 da Lei $n^{\circ}$ 10.696, de 2 de julho de 2003, que institui o Programa de Aquisição de Alimentos, e o Capítulo III da Lei no 12.512, de 14 de outubro de 2011, e dá outras providências. Diário Oficial da União, Brasília, DF, 4 de julho de 2012. Disponível em: https://www.planalto.gov.br/ccivil_03/_ato2011-2014/2012/decreto/d7775.htm. Acesso em: 01 out. 2014.

CAMARGO, R. A. L.; BACCARIN, J. G.; SILVA, D. B. P. O papel do Programa de Aquisição de Alimentos (PAA) e do Programa Nacional de Alimentação Escolar (PNAE) no fortalecimento da agricultura familiar e promoção da segurança alimentar. Temas de Administração Pública, v. 8, n. 2, p. 1-21, 2013.

CHARNES, A.; COOPER, W.W.; RHODES, E.

Measuringtheefficiencyofdecisionmakingunits. EuropeanJournalofOperationalRese arch, Amsterdam, v. 2, n. 6, p. 429-444, 1978.

CHMIELEWSKA, D.; SOUZA, D.; LOURETE, A. A. O Programa de Aquisição de Alimentos da Agricultura Familiar (PAA) e as práticas dos agricultores participantes orientadas ao mercado: estudo de caso no Estado de Sergipe. Brasília: IPEA, 2010 (Texto para Discussão, 1510). 
COELLI, T. J. A guide to DEAP version 2.1: a data envelopment analysis program. Armidale, Austrália: University of New England, 1996. p. 49. (CEPA Working Papers, 08/96).

CONAB - Companhia Nacional de Abastecimento. Programa de Aquisição de

Alimentos: resultados das ações da CONAB em 2015. Brasília: CONAB, 2016. 24 f. Disponível em:

http://www.conab.gov.br/OlalaCMS/uploads/arquivos/16_02_02_16_00_42_acoes_d a_conab_paa_2015.pdf. Acesso em: 01 ago. 2016.

CORREIO BRAZILIENSE. Assentados do Distrito Federal são exemplos na venda de produtos para o PAA. 2014. Disponível em:

http://www.correiobraziliense.com.br/app/noticia/cidades/2014/02/08/interna_cidades df,411876/assentados-do-distrito-federal-sao-exemplos-na-venda-de-produtos-parao-paa.shtml. Acesso em: 20 jan. 2016.

DELGADO, G. C.; CONCEIÇÃO, J. C.; OLIVEIRA, J. J. Avaliação do programa de aquisição de alimentos da agricultura familiar. Brasília: IPEA, 2005.

DEVES, O. D. Fortalecimento da agricultura familiar através do Programa de Aquisição de Alimentos - PAA: o caso do município de São Pedro do Butiá-RS. 2009. 157 f. Dissertação de Mestrado (Desenvolvimento Rural). Universidade Federal do Rio Grande do Sul, Faculdade de Ciências Econômicas, Programa de Pós-Graduação em Desenvolvimento Rural. Porto Alegre, 2009.

DIAS, T. F.; NUNES, E. M.; TORRES, F. L.; TORRES, A. C. M. O Programa de Aquisição de Alimentos da Agricultura Familiar (PAA) como estratégia de inserção socioeconômica: o caso do Território da Cidadania Sertão do Apodi (RN). Revista

Brasileira de Gestão e Desenvolvimento Regional, Taubaté, SP, v. 9, n. 3, p. 100$129,2013$.

GOMES, E. G. Uso de modelos DEA em agricultura: revisão da literatura.

Engevista, Niterói, v. 10, n. 1, p. 27-51,2008.

GRISA, C.; SCHMITT, C. J.; MATTEI, L. F.; MALUF, R. S.; LEITE, S. P. Contribuições do Programa de Aquisição de Alimentos à segurança alimentar e nutricional e à criação de mercados para a agricultura familiar. Agriculturas, Rio de Janeiro, v. 8, n. 3, p. 34-41, 2011.

GRISA, C.; SCHMITT, C. J.; MATTEI, L. F.; MALUF, R. S.; LEITE, S. P. O Programa de Aquisição de Alimentos (PAA) em perspectiva: apontamentos e questões para o debate. Retratos de Assentamentos, n. 13, p. 137-170, 2010.

HESPANHOL, R.A.M. O Programa Nacional de Fortalecimento da Agricultura Familiar - PRONAF: uma avaliação preliminar. Caderno Prudentino de Geografia, Presidente Prudente, n.22, p. 41-59, 2000.

IBGE - Instituto Brasileiro de Geografia e Estatística. Censo agropecuário de 2006. 2006. Disponível em:

http://www.sidra.ibge.gov.br/bda/pesquisas/ca/default.asp?o=2\&i=P. Acesso em: 10 out. 2014. 
JUBRAN, A. J. Modelo de análise de eficiência na administração pública: um estudo aplicado às prefeituras brasileiras usando a análise envoltória de dados. 2006. 226 f. Tese (Doutorado em Engenharia). Escola Politécnica, Universidade de São Paulo, São Paulo, 2006.

KASSAI, S. Utilização da análise envoltória de dados (DEA) na análise de demonstrações contábeis. 2002. $350 \mathrm{f}$. Tese (Doutorado em Contabilidade e Controladoria). Faculdade de Economia, Administração e Contabilidade, Universidade de São Paulo, São Paulo, 2002.

LEMOS, S. M.; LIMA, I. S. O programa de aquisição de alimentos (PAA) como política pública para a agricultura familiar e o desenvolvimento local. Oikos, Viçosa, v. 25, n. 1, p. 69-92, 2014.

LOPES, D. E; ALMEIDA, R. A. A. Avaliação do programa de aquisição de alimentos (PAA) e do programa nacional de alimentação escolar (PNAE) nos municípios de Castilho e Andradina-SP. Revista Pegada Eletrônica, v. 13, n.1, p. 132-148, 2012.

MAGALHÃES, K.A; CAMPOS, R.T. Eficiência técnica e desempenho econômico de produtores de leite no Estado do Ceará, Brasil. Revista de Economia e Sociologia Rural, Rio de Janeiro, v. 44, n. 4, p. 695-711, 2006.

MARINHO, A. Estudo de eficiência em alguns hospitais públicos e privados com a geração de rankings. Texto para discussão n. 794. Rio de Janeiro: IPEA, 2001.

MARQUES, P. E. M.; MOAL, M. F. L.; ANDRADE, A. G. F. de. Programa de Aquisição de Alimentos (PAA) no Estado de São Paulo: agricultura de proximidade em questão. Ruris, Campinas, v.8, n.1, p.63-89, 2014.

MDS - Ministério do Desenvolvimento Social e Combate à Fome. 2011. PAA data. Disponível em:

http://aplicacoes.mds.gov.br/sagi/paa/2011/visi_paa_conab/pg_principal.php. Acesso em: 10 out. 2014.

MDS - Ministério do Desenvolvimento Social e Combate à Fome. Estudo comparativo das diferentes modalidades do PAA-Região Nordeste. Brasília, Março de 2006. Disponível em

http://aplicacoes.mds.gov.br/sagi/PainelPEI/Publicacoes/Estudo\%20comparativo\%20 das\%20diferentes\%20modalidades\%20do\%20PAA-Regiao\%20Nordeste.pdf. Acessado em: 18 ago. 2015.

MEZA, L.A.; BIONDI NETO, L.; MELLO, J.C.C.B.S.; GOMES, E.G. ISYDIS Integrated system for decision support (SIAD - Sistema Integrado de Apoio à Decisão): a software package for data envelopment analysis model. Pesquisa Operacional,Niterói, v. 25, n. 3, p. 493-503, 2005.

MONTEIRO, T. M. A avaliação dos impactos sociais dos programas de governo. In: VII Congreso Internacional del CLAD sobre la Reforma del Estado y de la Administración Pública, Lisboa, Portugal, p. 8-11, 2002. 
MULLER, A. L.; SILVA, M. K.; SCHNEIDER, S. A construção das políticas públicas para a agricultura familiar no Brasil: o Programa de Aquisição de Alimentos. Estudos Sociedade e Agricultura, Rio de Janeiro, v. 20, n. 1, p. 106-138, 2012.

PAULA, M. M.; KAMIMURA, Q. P.; SILVA, J. L. G. Mercados institucionais na agricultura familiar: dificuldades e desafios. Revista de Política Agrícola, Brasília, v.23, n.1, p. 33-43, 2014

PEDROSO, M. M.; CALMON, P. C. P.; BANDEIRA, L. F. O uso da Análise Envoltória de Dados para avaliação da gestão do Programa Bolsa Família. Comunicação em Ciências da Saúde. v. 20, n.1, p. 37-44, 2009.

PEÑA, C. R. Um modelo de avaliação da eficiência da administração pública através do método Análise Envoltória de Dados (DEA). RAC-Revista de Administração Contemporânea, Curitiba, v. 12, n. 1, p. 83-106, 2008.

PLEIN, C.; FILIPPIN, E. E. O Programa Aquisição de Alimentos da Agricultura Familiar (PAA): geração de renda e segurança alimentar. Faz Ciência, Francisco Beltrão, v. 15, n. 20, p.63-86, 2012.

REIS, P.R.C. Política pública de previdência social e nível de bem-estar: impacto sobre as famílias e municípios de Minas Gerais. Dissertação (Mestrado em Administração), Universidade Federal de Viçosa, Viçosa-MG, 2012.

RICHARDSON, R. J. Pesquisa social: métodos e técnicas. 3. ed. São Paulo: Atlas, 1999.

SAGRILO, E. (Ed.).Agricultura familiar. Teresina: Embrapa Meio-Norte. 2002. 74 p. (Embrapa Meio-Norte, Sistemas de Produção, 1). Disponível em: http://www.cpamn.embrapa.br/publicacoes/new/sistemaproducao/sistemaproducao_ pdf/sistemaproducao_1.pdf. Acesso em: 10 Dez. 2014.

SANT'ANA, A. L.; SILVA, F. C.; MODENESE, V. S.; SANT'ANA, D. Z.; SOUZA, G. S. Qualidade partilhada e relações de sociabilidade entre produtores familiares e consumidores no processo de comercialização direta, no noroeste paulista. In:Mercados e Agricultura Familiar: interfaces, conexões e conflitos / Marcelo Antonio Conterato... [et al.] (Org.). Porto Alegre: Via Sapiens, 2013. 358 p.: il. (Difusão IEPE/UFRGS). p. 133-154.

SIMÃO, G. L; SILVA, E. A; SILVEIRA, S. F. R. Grau de cobertura do Programa de Aquisição de Alimentos (PAA) junto aos agricultores familiares do estado de Minas Gerais. Revista de Economia e Sociologia Rural, v.52, n.3, p. 533-548, 2014.

SOUZA, C. Políticas Públicas: uma revisão da literatura. Sociologias, Porto Alegre, Ano 8, n. 16, p. 20-45. Jul./dez. 2006.

SOUZA, P.C.T.; WILHELM, V.E. Uma introdução aos modelos DEA de eficiência técnica. Tuiuti, Curitiba, n. 42, p. 121-139, 2009.

SPAROVEK, G.; PLATA, L. A. E.; MAULE, R. F.; MAULE, F. E.; KLUG, F. S. F.; KLUG, I. L. F.; GOLDSZMIDT, R.; FERNANDES, R. L.; CAMARGO, R. A.;

MARTINS, S. P. Estudo comparativo das diferentes modalidades do PAA: região 
Nordeste. In: PAES-SOUZA, R.; VAITSMAN, J. Cadernos de estudo:

desenvolvimento social em debate - síntese das pesquisas de avaliação de programas sociais do MDS, n. 5, 2007. p. 62-64.

TEIXEIRA, E. C. O papel das políticas públicas no desenvolvimento local e na transformação da realidade. Salvador, Bahia: AATR, 2002.

VALNIER, A.; RICCI, F. Programa de Aquisição de Alimentos (PAA): uma análise comparativa nos estados de Rondônia e Acre. Campo-Território, Uberlândia, v. 8, n. 16, p. 198-228, 2013. 